\title{
Evidence of attraction between charge carriers in a doped Mott insulator
}

\author{
Emil Blomquist ${ }^{1}{ }^{1}$ and Johan Carlström ${ }^{2}$ \\ ${ }^{1}$ Department of Physics, Royal Institute of Technology, SE-106 91 Stockholm, Sweden \\ ${ }^{2}$ Department of Physics, Stockholm University, SE-106 91 Stockholm, Sweden
}

(Received 12 August 2020; revised 16 December 2020; accepted 25 February 2021; published 23 March 2021)

\begin{abstract}
Recent progress in optically trapped ultracold atomic gases is now making it possible to access microscopic observables in doped Mott insulators, which are the parent states of high-temperature superconductors. This makes it possible to address longstanding questions about the temperature scales at which attraction between charge carriers are present, and their mechanism. Controllable theoretical results for this problem are not available at low temperature due to the sign problem. In this work, we make important progress with this problem by employing worm-algorithm Monte Carlo, which allows us to obtain completely unbiased results for two charge carriers in a Mott insulator. Our method gives access to lower temperatures than what is currently possible in experiments, and provides evidence for attraction between dopants at a temperature scale that is now feasible in ultracold atomic systems. We also report on spin correlations in the presence of charge carriers, which are directly comparable to experiments.
\end{abstract}

DOI: 10.1103/PhysRevResearch.3.013272

Ultracold atomic gases have provided an alternative path to exploring the physics of high-temperature superconductors by emulation of a doped Mott insulator. This system, with charge carriers propagating on an antiferromagnetic background, is widely regarded as the parent state of high-temperature superconductivity [1]. At low doping, charge carriers form quasiparticles known as polarons, as a result of competition between kinetic energy and superexchange processes. The carrier can reduce its kinetic energy by delocalizing, but this naturally distorts the spin background. Minimizing the superexchange energy leads to a state with strong antiferromagnetic correlations, which increases the kinetic energy. The polaron emerges as the best compromise. Increasing the doping, the system gradually evolves into a Fermi liquid. Recent experiments on ultracold atomic gases indicate that this crossover is completed at a doping of approximately $30 \%$ [2].

It is generally believed that pairing in the high-temperature superconductors occurs as a result of an attractive interaction between charge carriers that is mediated by the spin background. The precise nature of the microscopic mechanism remains debated, however. A circumstance which complicates this question is that superconductivity does not emerge from a conventional metallic state, but rather from a non-Fermi liquid [1].

The search for a pairing mechanism has lead to considerable interest in how doping alters spin correlations in Mott insulators. For example, the resonating valence bond (RVB) theory claims that the spins form a superposition of singlets, providing a better compromise between kinetic and magnetic

Published by the American Physical Society under the terms of the Creative Commons Attribution 4.0 International license. Further distribution of this work must maintain attribution to the author(s) and the published article's title, journal citation, and DOI. energy [3]. If such a mechanism is at play, then it should have implications for the spin background, possibly even above the onset of superconductivity.

Another point of contention is whether fermions form pairs before the onset of superconductivity, such that these merely condense at the critical temperature [4,5]. The conjecture of preformed pairs seems to be invited by the scale of the super-exchange parameter $J$, which in the high-temperature superconductors corresponds to approximately $1500 \mathrm{~K}[6,7]$. This raises questions about the temperature range where attraction between charge carriers can be observed. In light of these considerations, spin and charge correlations have become the focal point of ultracold-atoms experiments.

Despite intense research, both the pairing mechanism and the nature of the normal state remain open questions, chiefly as a consequence of a lack of theoretical methods that can address strongly correlated fermions with sufficient accuracy. The Hubbard model—which is believed to capture the essential physics of cuprates - has been investigated with a number of approximative many-body techniques [8], including density-matrix renormalization-group theory (DMRG) [9], dynamic mean-field theory [10-15], and auxiliary field quantum Monte Carlo [16-19]. These methods are able to reproduce several states that are believed to be relevant for cuprate superconductors, including antiferromagnetism, stripes, pseudogap physics, and $d$-wave superconductivity. However, upon comparison, the aforementioned techniques display notable discrepancies when it comes to predicting the ground state of the Hubbard/ $t-J$ models [20]. This suggests that strongly correlated systems may be highly sensitive to uncontrolled approximations or discretization.

To date, there are few unbiased numerical techniques for strongly correlated fermions. Numerical linked cluster expansion has provided the equation of state as well as spin correlations to exceptional precision [21,22], but has thus far not given any insights into polaron physics or superconductivity. 
Diagrammatic Monte Carlo techniques [23] can resolve pairing in the Fermi-liquid regime [24]. Recently, strides have been made to advance this method into the regime of strong interactions [25-30]. However, as it stands, currently published results are limited either to comparatively high temperatures or to parameter regimes where the onsite repulsion is smaller than the band width. For small systems, exact diagonalization and the Lanczos technique do provide indications of attraction between charge carriers [31].

The lack of theoretical methods for correlated fermions has motivated an intense effort to develop experimental techniques with access to microscopic observables. With the advent of quantum gas microscopy, imaging of entangled many-body states is now possible at the level of single-site resolution [32]. Using ultracold atomic gases, Mott-insulating states can be created and cooled to the point where antiferromagnetic correlations become significant [33].

Recent experimental work has focused on spin-correlations in doped systems $[34,35]$. In particular, this has resulted in the first observation of the internal structure of a polaron on an antiferromagnetic background, revealing microscopic details about the cloud of spins surrounding the carrier [36]. The search for correlations between dopants and thus signs of effective interactions has also been initiated [37].

\section{METHODS}

In this work, we use worm-algorithm Monte Carlo (WAMC), introduced in [38], to extract completely unbiased charge and spin correlations for a pair of holes in a Mott insulator, in the presence of thermal fluctuations. Our findings indicate that attraction between carriers is present at an energy scale corresponding to approximately $700 \mathrm{~K}$, significantly above the critical temperature. This is a regime that could realistically be achieved in ultracold atomic gases in the near future. We also report on spin correlations in the presence of two interacting dopants, finding that the delocalization of multiple carriers can explain the reversal of correlators seen in recent experiments [35,37].

WAMC is a technique that allows extremely efficient sampling of world lines, from which we obtain diagonal elements of the density matrix at thermal equilibrium. Our observables are therefore not subject to any bias, since they are exactly represented by the distribution of world lines. This sets our method apart from previous works, which are either based on uncontrolled approximations, or are confined to small systems, where finite size effects are considerable. WAMC is mainly applied to bosons due to the fermionic sign. However, for Gutzwiller-projected theories, the sign problem is only extensive in the number of charge carriers as opposed to the system size. Using a highly efficient sampling protocol, it is possible to address a small number of dopants in a system that is sufficiently large to avoid finite-size effects. Previously, this method has been used to extract spectral properties of a single carrier [39]. More recently, it has also provided the real-space structure of a single polaron [40]. For a discussion of the sign problem in WAMC, see the Appendix.

In this work, we represent charge carriers and spins as world lines in space and imaginary time. We use a $20 \times 20$ lattice with periodic boundary conditions to prevent finite-

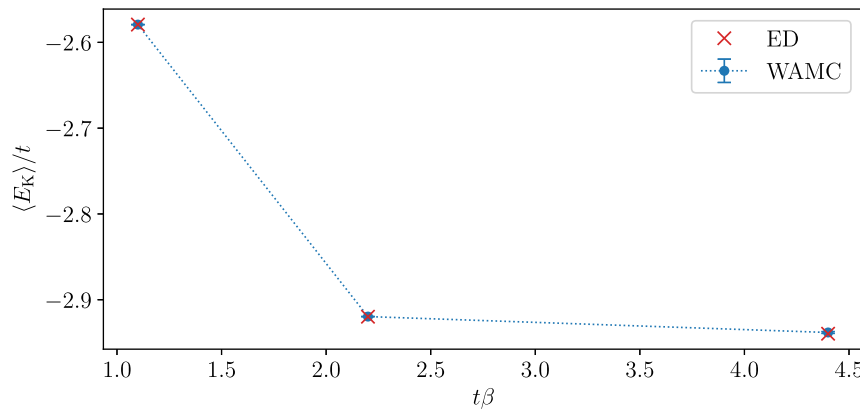

FIG. 1. Comparison between worm-algorithm Monte Carlo and exact diagonalization. Computing the kinetic energy for a single carrier in a $4 \times 4$ system using the worm algorithm, we can benchmark it against exact diagonalization. As expected for an unbiased technique, we obtain perfect agreement. Here, $t / J=10 / 3$.

size effects. We use separate worms for the spin and charge sectors [38]. The former can wind in imaginary time, which alters the total spin in the system so that this sector is in the grand canonical ensemble. The worm corresponding to charge cannot wind, keeping the number of carriers to two at all times (in the partition function sector). By generating contributions to the trace of the density matrix at thermal equilibrium, we obtain access to essentially arbitrary operator expectation values, including spin and charge correlators. To confirm the accuracy of this method, we provide benchmarks against exact diagonalization; see Fig. 1 and also the Appendix.

We describe the system using the $t-J$ model [41]

$$
\begin{aligned}
\hat{H}= & -t \sum_{\langle i j\rangle, \sigma} \hat{c}_{i \sigma}^{\dagger}\left(1-\hat{n}_{i \bar{\sigma}}\right) \hat{c}_{j \sigma}\left(1-\hat{n}_{j \bar{\sigma}}\right) \\
& +J \sum_{\langle i j\rangle}\left(\hat{\mathbf{S}}_{i} \cdot \hat{\mathbf{S}}_{j}-\frac{\hat{n}_{i} \hat{n}_{j}}{4}\right), \quad \bar{\sigma}=-\sigma,
\end{aligned}
$$

which effectively captures the low energy physics of the Hubbard model [42] in the case of large onsite repulsion. Here $\hat{c}_{i \sigma}, \hat{c}_{i \sigma}^{\dagger}$ denote annihilation and creation operators of a spin $\sigma$ electron at the site $i$. The total particle number on the site $i$ is given by $\hat{n}_{i}=\sum_{\sigma} \hat{n}_{i \sigma}$ where $\hat{n}_{i \sigma}=\hat{c}_{i \sigma}^{\dagger} \hat{c}_{i \sigma}$. Two energy scales thus describe the model (1). The kinetic energy is due to hole propagation, and is proportional to $t$. The superexchange energy originates in the virtual creation and annihilation of doublon-hole pairs and is proportional to $J=4 t^{2} / U$, where $U$ is the onsite repulsion.

In the low mobility limit, where $t / J \rightarrow 0$, the attraction between two carriers can be understood from a simple broken-bond picture: An isolated hole negates four superexchange interactions. When two carriers share a link, the number of broken bonds is reduced from 8 to 7 , saving some magnetic energy. For realistic model parameters, where $t / J$ is not vanishing, this picture becomes overly crude, however. Binding the two holes together impairs delocalization, resulting in competition between kinetic and exchange-mediated attraction. Therefore, it is expected that the binding energy decreases with increasing $t / J$, to possibly vanish at some critical value $(t / J)_{c}$ [43]. This picture is corroborated by zero-temperature calculations on small clusters based on the Lanczos algorithm, where it is found that with 

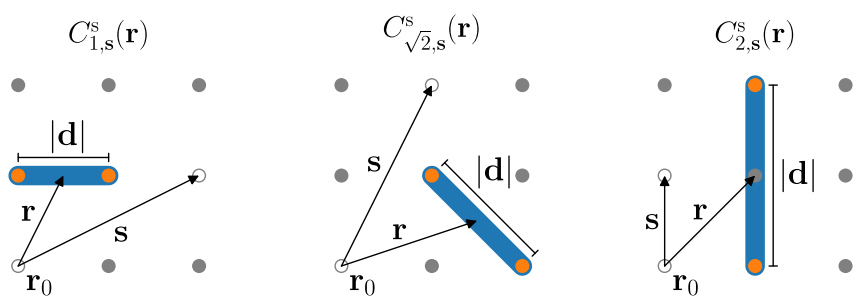

FIG. 2. Illustration of spin-spin correlators. The spin-spin correlators $C_{|\mathbf{d}|, \mathbf{s}}^{\mathrm{s}}(\mathbf{r})$ defined in Eq. (A2) illustrated in the cases of $|\mathbf{d}|=1$, $|\mathbf{d}|=\sqrt{2}$, and $|\mathbf{d}|=2$. Here, the bond distance $\mathbf{r}$ is defined as the distance from one of the carriers to the point between the spins for which the correlation is considered and $\mathbf{s}$ is the distance to the second carrier.

increasing $t / J$, the binding energy decreases, while the typical separation grows. However, being limited to systems of up to 26 lattice sites, these results cannot resolve bound pairs where the distance between carriers is large compared to the system size [31]. DMRG calculations on notably larger clusters-up to $10 \times 7$ sites-also indicate attraction at zero temperature [44].

At realistic values of $t / J$, cluster calculations give a relatively small amplitude for finding the holes on neighboring sites, stressing the inadequacy of the broken-bond model [31]. Meanwhile, the delocalization of a charge carrier gives rise to frustration due to competition between kinetic and magnetic processes. This has motivated the suggestion that frustrated bonds may mediate attraction between carriers [44].

To obtain indications of attraction between charge carriers that are directly comparable to experiments, we calculate the hole-hole correlator-in a frame of reference where one hole is always located at the origin — which takes the form

$$
C^{\mathrm{h}}(\mathbf{s})=(N-1)\left\langle\hat{n}_{\mathbf{0}}^{\mathrm{h}} \hat{n}_{\mathbf{s}}^{\mathrm{h}}\right\rangle-1 .
$$

Here $\hat{n}_{\mathbf{r}}^{\mathrm{h}}=1-\hat{n}_{\mathbf{r}}$, is the hole-number operator at site $\mathbf{r}, \mathbf{s}$ is the distance between the two holes, and $N$ is the number of lattice sites. Since the spin background is expected to mediate attraction between carriers, we also calculate spin-correlations of the form

$$
C_{|\mathbf{d}|, \mathbf{s}}^{\mathrm{s}}(\mathbf{r})=4 \frac{\sum_{\mathbf{r}_{0}}\left\langle\hat{n}_{\mathbf{r}_{0}}^{\mathrm{h}} \hat{n}_{\mathbf{r}_{0}+\mathbf{s}}^{\mathrm{h}} \hat{S}_{\mathbf{r}_{0}+\mathbf{r}-\mathbf{d} / 2}^{z} \hat{S}_{\mathbf{r}_{0}+\mathbf{r}+\mathbf{d} / 2}^{z}\right\rangle}{\sum_{\mathbf{r}_{0}}\left\langle\hat{n}_{\mathbf{r}_{0}}^{\mathrm{h}} \hat{n}_{\mathbf{r}_{0}+\mathbf{s}}^{\mathrm{h}}\right\rangle} .
$$

Here $\mathbf{r}_{0}$ and $\mathbf{r}_{0}+\mathbf{s}$ are the positions of the two carriers, and similarly $\mathbf{r}_{0}+\mathbf{r} \pm \mathbf{d} / 2$ are the positions of the two spins, located a distance $\mathbf{d}$ from one another. We will consider the cases $|\mathbf{d}|=1$ (nearest neighbor), $|\mathbf{d}|=\sqrt{2}$ (next-nearest neighbor), and $|\mathbf{d}|=2$ (next-next-nearest neighbor). These correlators are illustrated in Fig. 2.

\section{RESULTS}

In Fig. 3, we show examples of hole-hole correlations for the cases $t / J=2$ and $t / J=5 / 3$. These reveal an attraction between the carriers which is manifested in a peak in the correlator $C^{\mathrm{h}}$ [Eq. (A1)] at small separation.

The scenario when $t / J=2$ corresponds to $U=8 t$ in the Hubbard model, i.e., where the onsite repulsion is equal to the band width. For this parameter set we observe a peak in $C^{\mathrm{h}}$
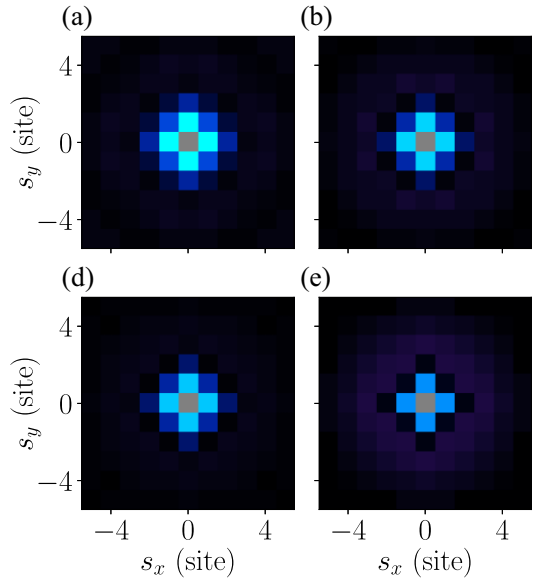

(e)
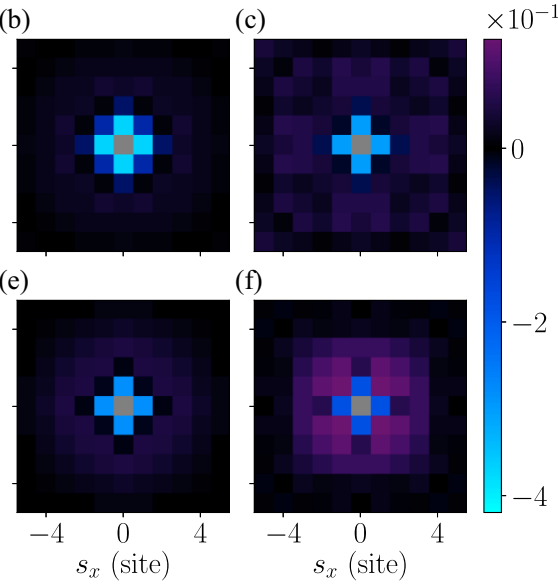

(f)
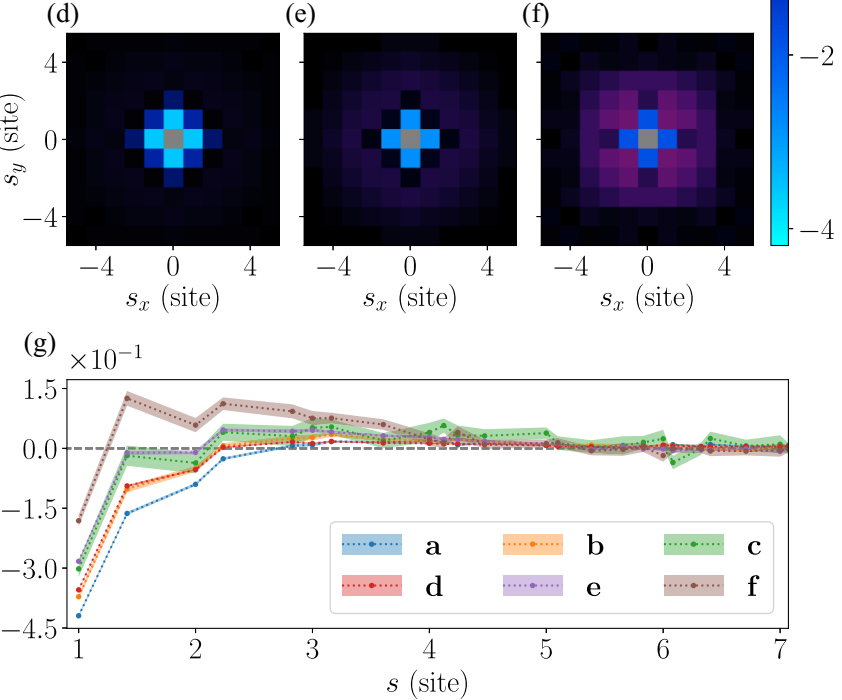

FIG. 3. Hole-hole correlations. (a)-(c) show contour plots of the correlator $C^{\mathrm{h}}(\mathbf{s})$ [Eq. (A1)] for the case $t / J=2$ and $\beta J=$ 2.1, 2.4, 2.7 respectively. The radial components of these are given in $(\mathrm{g})$. For this parameter set, we observe a peak in the probability distribution at small separation which is visible already at temperatures corresponding to $J \beta=2.1$. In (a) the effect is small, with a peak value of $C^{\mathrm{h}} \approx(2.3 \pm 0.27) \times 10^{-2}$ at $s \approx 4.12$. Decreasing the temperature bolsters the effect, and in (c) we observe a maximum value of $C^{\mathrm{h}} \approx(6.0 \pm 1.7) \times 10^{-2}$, also at a separation of $s \approx 4.12$. This scenario corresponds to $U=8 t$ in the Hubbard model, which is the parameter set that was realized in the experiment [37]. Slightly decreasing $t / J$ increases the height of the peak of $C^{\mathrm{h}}$. In (d)-(f), contour plots of $C^{\mathrm{h}}(\mathbf{s})$ are given for $t / J=5 / 3$ and $\beta J=2.1,2.4,2.7$ respectively. The corresponding radial components are again shown in (g). At the lowest temperature (f), the peak in $C^{\mathrm{h}}$ is found when the holes are next-nearest neighbors.

which is present at $J \beta=2.1$ [Fig. 3(a)]. For comparison, the energy scale of the superexchange in cuprate superconductors has been estimated to $J=128 \pm 5 \mathrm{meV}$, corresponding to approximately $1500 \mathrm{~K}$. This suggests an onset of weak attraction at a temperature equivalent to approximately $700 \mathrm{~K}$, though it should be stressed that the ratio of the kinetic and magnetic energy scales in the cuprates is somewhat larger, with estimates at $t / J \approx 3.3[6,7]$. This parameter set was recently realized in an optically trapped ultracold atomic gas. Examination of charge correlations at the temperature $J \beta \approx 1.53 \mathrm{did}$ not reveal any signs of attraction between carriers, consistent with our results [37].

The fact that attraction is only observed at a sufficiently low temperature scale suggests that the effective interaction between carriers is altered by thermal fluctuations. Indeed, zero-temperature estimates of the binding energy obtained from the Lanczos algorithm and DMRG give results in the span $0.6 \geqslant \epsilon_{\text {binding }} / t \geqslant 0.15$ when $t / J=2$. In a temperature 


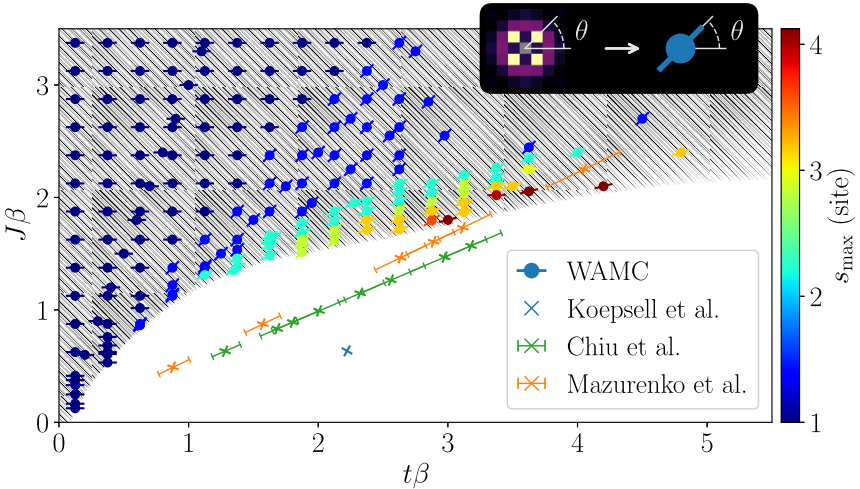

FIG. 4. Parameter regions with attraction. Discs denote parameters for which the correlator $C^{\mathrm{h}}$ exhibits a peak at a carrier separation $\mathbf{s}_{\max }$, which indicates attraction. The bar intersecting the disk gives the orientation of $\mathbf{s}_{\max }$, while the color gives its magnitude according to the legend. The shaded region is a guide to the eye. For comparison, we also indicate the parameter sets of three recent experiments. In Koepsell et al. [36], the internal structure of a single polaron was mapped out using quantum gas microscopy. In Chiu et al. [37], correlations between dopants in an ultracold atomic gas were examined. In Mazurenko et al. [33], spin correlations in the Hubbard model were examined, though this parameter set, strictly speaking, does not correspond to a Mott insulator.

range where $\beta t=4.2-5.4$, this energy scale would be expected to impose significant correlations. The fact that these remain small, only reaching $C^{\mathrm{h}} \approx 6 \%$, suggests that the interactions between charge carriers are affected by thermal fluctuations.

This can partially be attributed to the loss of the magnetic correlations that mediate attraction. However, it is also the case that spin fluctuations increase the delocalization of the carrier, and this may also work against pair formation. The latter effect is manifested in the fact that the kinetic energy of a dopant may actually increase as the temperature is lowered [40].

The fact that fluctuations alter interactions-even to the point where attraction vanishes-suggests that ground state results provide limited guidance regarding the temperature ranges where we can expect to observe attraction in ultracold atomic gases, where strong temperature effects are ubiquitous.

Decreasing the scale of the kinetic energy to $t / J=5 / 3$ suppresses delocalization. The peak in $C^{\mathrm{h}}$ sets in at $J \beta \approx 1.8$ and is now larger at comparable temperatures. Eventually, the correlator attains its maximum when the carriers are nextnearest neighbors [Fig. 3(f)].

By generating hole-hole correlations for a wide range of parameters and systematically testing whether $C^{\text {h }}$ possesses a maximum (within two standard deviations; see Appendix for details), we obtain the phase diagram shown in Fig. 4. To put this data into context, we have included the model parameters for three recent experiments. The polaron studied in [36] is situated far from the region of attraction, owing to a relatively high temperature and also large onsite repulsion. The experiment on correlations between dopants reported in [37] is, however, closer to the onset of attraction. For this parameter set, our results indicate that a peak in $C^{\mathrm{h}}$ appears (a)

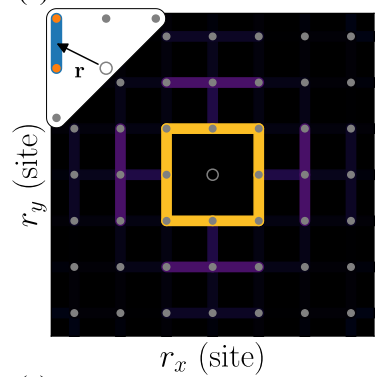

(c)

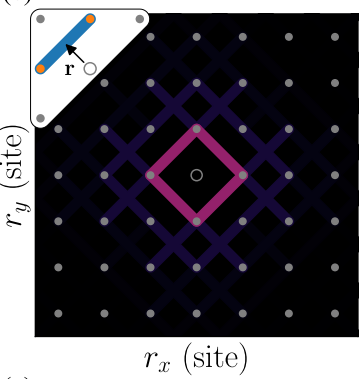

(e)

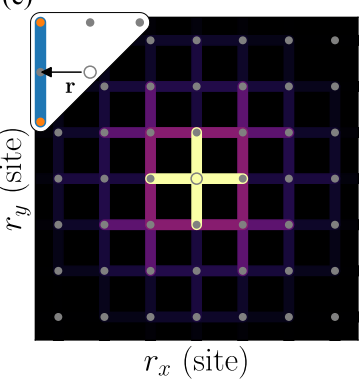

(b)
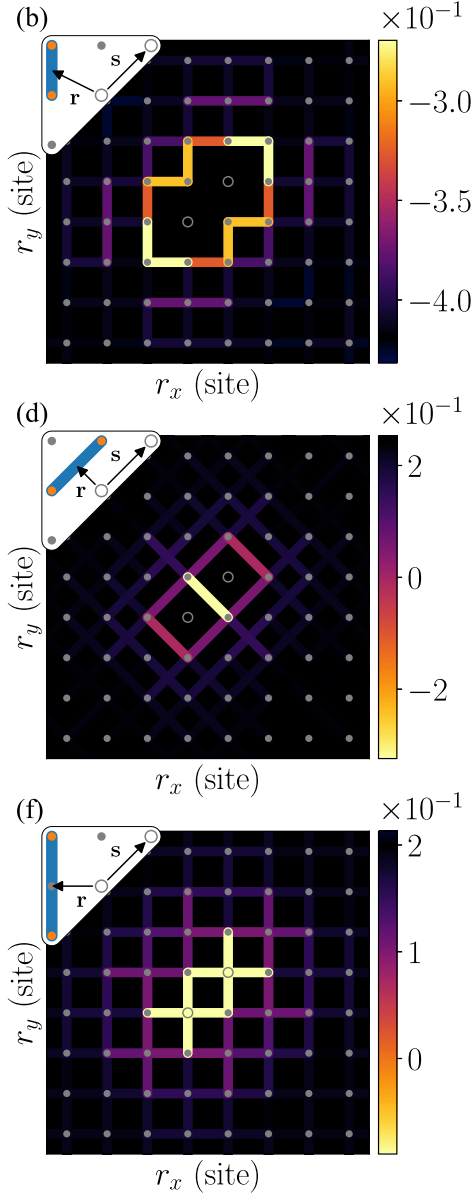

FIG. 5. Spin correlations $C_{|\mathbf{d}| \mathbf{s}}^{\mathrm{s}}(\mathbf{r})$ in the proximity of carriers. Left column depicts a single hole, while right column shows two dopants which are next nearest neighbors. The top row (a), (b) gives $C_{|\mathbf{d}|=1, \mathbf{s}}^{\mathrm{s}}(\mathbf{r})$, middle row (c), (d) gives $C_{|\mathbf{d}|=\sqrt{2}, \mathbf{s}}^{\mathrm{s}}(\mathbf{r})$, and bottom row (e), (f) gives $C_{|\mathbf{d}|=2, \mathbf{s}}^{\mathrm{s}}(\mathbf{r})$. Model parameters are $J \beta=2.4$ and $t / J=2$. Additional examples of spin-correlations are provided in the Appendix. Animations can be found here [46].

at $J \beta \approx 2.1$. Even lower temperatures are achievable, as exemplified by [33], were long-range spin-correlations were reported at $T / t \approx 0.25$. The phase diagram confirms that the attraction is sensitive to the ratio $t / J$. This is also the case at zero temperature, and stems from competition between delocalization and binding of the carriers [43].

In Fig. 5, we show spin-correlators [Eq. (A2)] for the case $t / J=2$ and $J \beta=2.4$, which is in the attractive regime. For a single carrier [panels (a), (c), and (e)] we observe a cloud of distorted spin-correlations as a result of competition between delocalization and superexchange, in line with $[36,40,45]$. This includes a suppression of nearest neighbor correlators (a) as well as the reversal of next-next-nearest neighbor correlations across the carrier (c).

In the proximity of the carrier, the next-nearest neighbor correlators (b) are strongly suppressed due to competing processes: When the dopant hops a single lattice spacing, spins that were previously next-nearest neighbors-and therefore would be correlated - are brought into direct contact, where the interaction is antiferromagnetic. This leads to a highly 
(a)

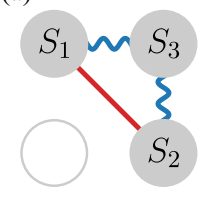

(d)<smiles></smiles>

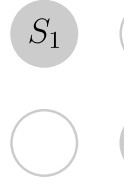

$\sim \mathrm{AF}$ interaction

- $/$ indirect $\mathrm{AF} / \mathrm{F}$ interaction

FIG. 6. Superexchange and kinetic-magnetic interaction. In the presence of a single hole (a), the spins $S_{1}$ and $S_{2}$ interact indirectly via super-exchange that is mediated by $S_{3}$. This gives rise to an effective ferromagnetic interaction. However, delocalization of the hole (a)-(c) brings $S_{1}$ and $S_{2}$ into direct contact, where interactions are antiferromagnetic. We refer to this as kinetic-magnetic interaction. The result is a high level of frustration and almost vanishing spin-correlations near the carrier. When two holes are present (d)-(f), only kinetic-magnetic interaction is present so that $S_{1}$ and $S_{2}$ become anticorrelated.

frustrated state with competition between a kinetic-magnetic interaction and superexchange; see Figs. 6(a)-6(c).

When two carriers are placed as next-nearest neighbors, the frustration is alleviated. The primary interaction between the two spins which are situated next to both dopants is now kinetic-magnetic [Figs. 6(d)-6(f)], causing remarkably strong anticorrelation as seen in Fig. 5(d). This result is consistent with magnetic properties, which have been observed in several recent experiments: When doping a Mott insulator, it is found that next-nearest neighbor spin correlations cross over from positive to negative at a carrier density of approximately $20 \%$ [35,37]. Notably, this crossover is not predicted by the structure of a single polaron, and the generation of strong anticorrelations must be understood as a multiple charge-carrier phenomenon. The alleviation of frustration has been identified as a possible source of attraction between carriers in doped Mott insulators [44].

\section{DISCUSSION}

In conclusion, we present high-precision data for spin and charge correlations in a Mott insulator with two dopants at finite temperature, obtained via worm-algorithm Monte Carlo. To the best of our knowledge, this is the first time that unbiased theoretical results are reported for this problem. Our findings indicate that the interactions are altered by thermal fluctuations, and that attraction sets in at $J \beta \approx 2.1$ when the onsite repulsion is equal to the bandwidth (i.e., $U=8 t$ ). This is a temperature range that is now experimentally feasible, and we argue that real progress in understanding this longstanding problem is now possible using optically trapped ultracold atoms and high-precision quantum Monte Carlo simulations in tandem.

Our technique gives access to lower temperatures than what is possible in experiments, allowing us to provide a phase diagram outlining parameter regions where attraction is present. We make detailed predictions about spin correlation in the proximity of the two carriers that are directly comparable to quantum gas microscopy and thus serve as a natural benchmark for future experiments.

\section{ACKNOWLEDGMENTS}

This work was supported by the Swedish Research Council (VR) through Grants No. 2018-03882, No. 642-2013-7837, and No. 2018-03659. It was also supported by Gålöstiftelsen, through a travel stipend. The computations were enabled by resources provided by the Swedish National Infrastructure for Computing (SNIC) at the National Supercomputer Centre in Linköping partially funded by the Swedish Research Council through Grant Agreement No. 2018-05973.

\section{APPENDIX}

\section{Worm-algorithm Monte Carlo}

Using a continuous-time worm-algorithm Monte Carlo (WAMC), we simulate a pair of holes in the $t-J$ model [41] on a quadratic lattice with $20 \times 20$ sites and periodic boundary conditions. The WAMC method effectively samples world-line configurations in real space and imaginary time by combining the partition function sector and Green's function sector: In order to go from one partition function world-line configuration to another, one needs to pass through the Green's function sector [38]. We use two different worms to represent spins and holes. The former is allowed to wind in imaginary time such that the total spin can vary. Contrarily, the hole worm is not, and the number of holes in the partition function sector is therefore kept fixed. All data presented are extracted purely from the sampled partition function configurations. In particular, the kinetic energy presented later in this Appendix is obtained by counting the kinks involving the hole worm [47].

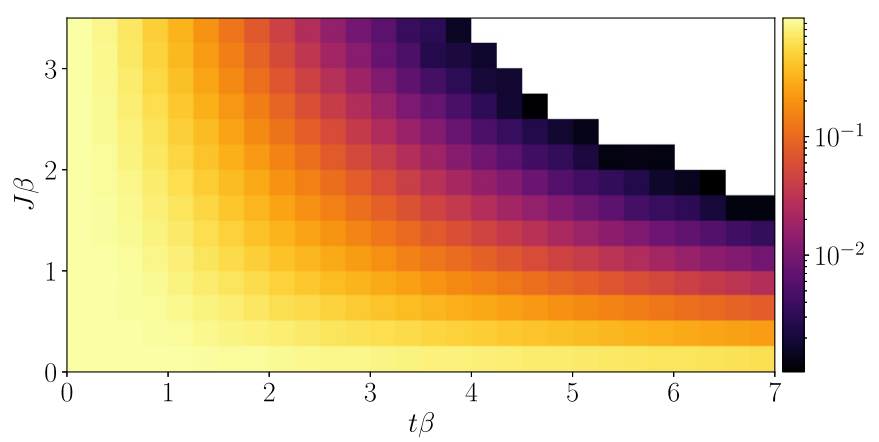

FIG. 7. Average sign. This plot shows the exponential decay of $\langle s\rangle^{\prime}$ with increased values of $t \beta$ and $J \beta$. Only values $\langle s\rangle^{\prime}>10^{-3}$ are shown due to an otherwise poor SNR. 
(a) $\times 10^{-1}$

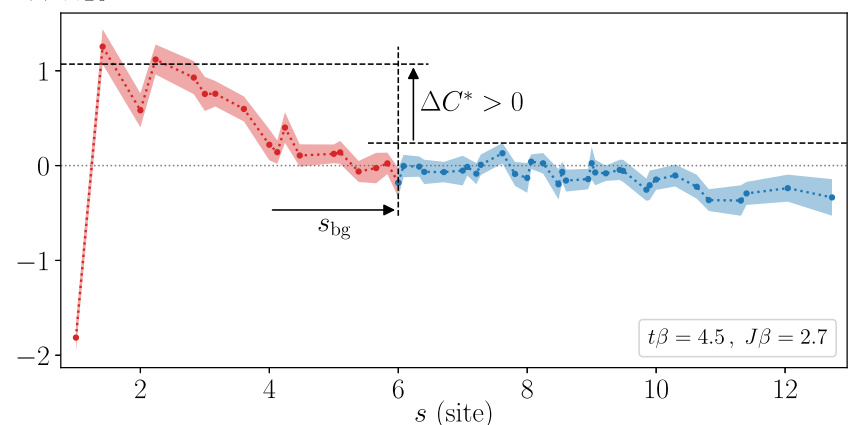

(b) $\times 10^{-1}$

(c)
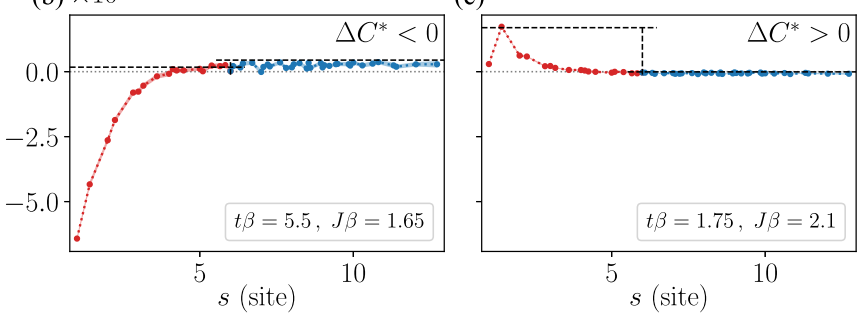

FIG. 8. Determining hole-hole attraction. Illustrations of how hole-hole attraction is inferred using radially projected hole-hole correlations obtained using WAMC. If the difference $\Delta C^{*}=$ $\max \left[C\left(s<s_{\mathrm{bg}}\right)\right]-\max \left[C\left(s \geqslant s_{\mathrm{bg}}\right)\right]-2 \times$ noise $>0$ [(a) and (c)], the data are said to indicate attraction, otherwise (b) attraction is deemed to be absent. The partition divider $s_{\mathrm{bg}}=6$ is chosen such that it exceeds separation distances where a peak is observed.

\section{Sign problem}

When simulating two holes in the $t-J$ model using WAMC, there are two types of processes contributing to the fermionic sign problem: exchange of indistinguishable spins and exchange of holes. The former enters first at order $t^{2} J^{3}$ [39] and the latter at order $t^{4}$. When such a process is present in a world-line configuration, the configuration weight $p$ might turn negative. Hence the name "sign problem."

In order to account for negative configuration weights in WAMC, we factor the weight $p$ into its sign $s= \pm 1$ and

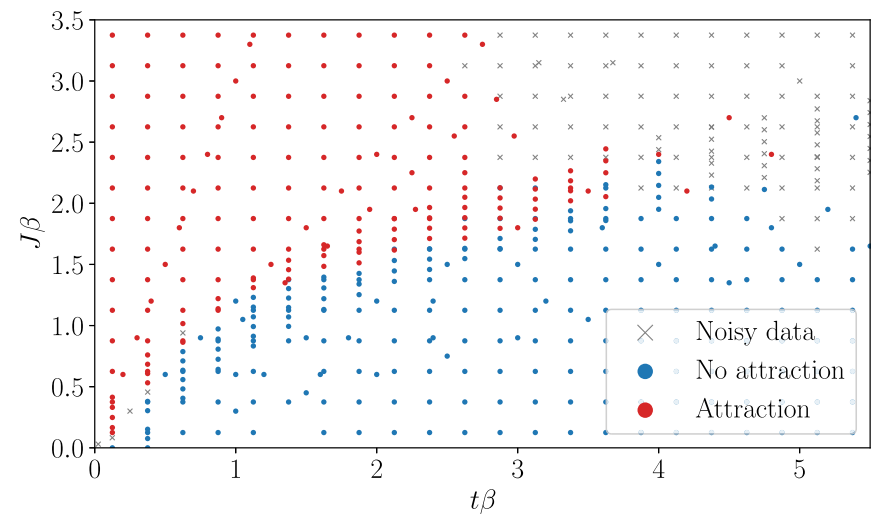

FIG. 9. Attractive parameter region. The parameter region is scanned using WAMC. Red dots indicate observed hole-hole attraction, blue dots signify no observed attraction, and gray crosses mark points deemed to have inadequate SNR. The reason for large parameter values suffering from a poor SNR can be explained by the sign problem (cf. Fig. 7). On the other hand, the low SNR for small parameter values is caused by a weak signal.

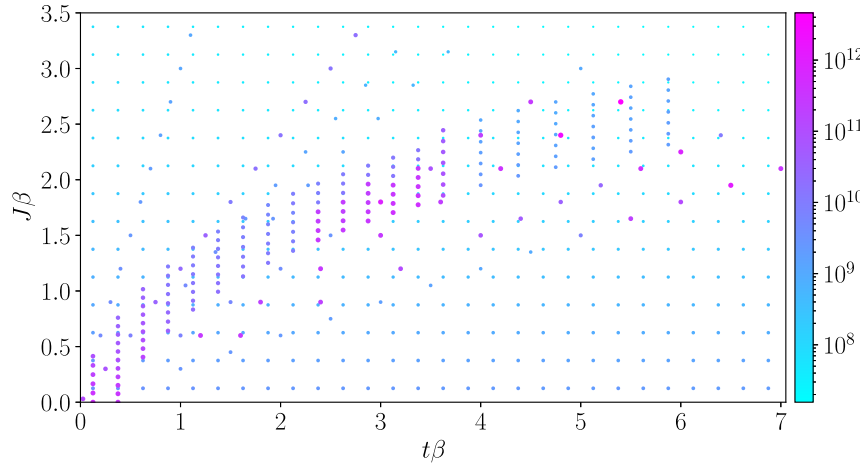

FIG. 10. Extent of simulation. This figure illustrates the number of sampled world-line configurations in the partition function sector for different values of $t \beta$ and $J \beta$. The combined total simulation time amounts to several million core-hours.

magnitude $|p|$, i.e., $p=s|p|$. Then, when computing the acceptance ration, $p$ is exchanged in favor of $|p|$, and every sampled quantity $x$ is assigned the sign $s$ of the world-line configuration. By performing this substitution, the acquired expectation value $\langle x\rangle^{\prime}$ is not that of the original fermionic system. However, the latter one is obtained through $\langle x\rangle=$ $\langle s x\rangle^{\prime} /\langle s\rangle^{\prime}$ [48]. This way of circumventing negative configuration weights gives rise to an exponential decrease in signal-to-noise ratio (SNR) and is the hallmark of the sign problem. The decrease of SNR can, in part, be explained by the exponential decay of the denominator $\langle s\rangle^{\prime}$ with, in our case, increased values of $t \beta$ and $J \beta$. This decay of $\langle s\rangle^{\prime}$ is illustrated in Fig. 7.

\section{Determining hole-hole attraction}

The starting point for determining hole-hole attraction is the hole-hole correlator, which in a frame of reference where
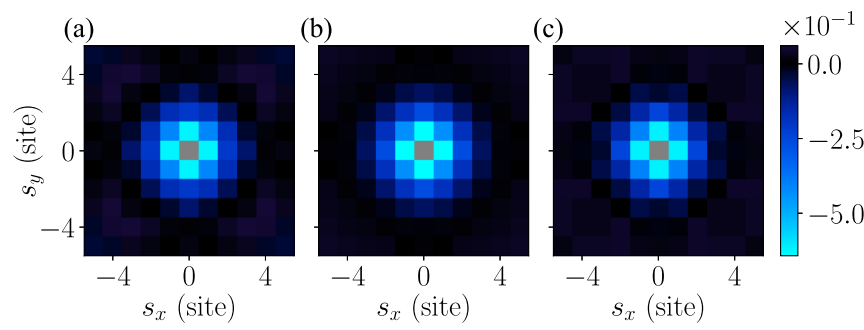

(d) $\times 10^{-1}$

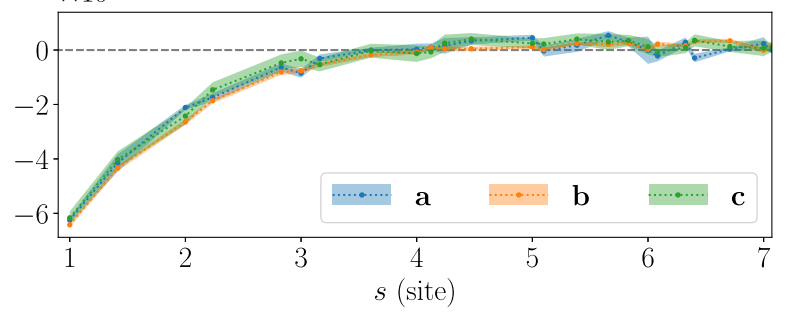

FIG. 11. Hole-hole correlations. (a)-(c) show contour plots of the correlator $C^{\mathrm{h}}(\mathbf{s})$ for the case $t / J=10 / 3$ and $\beta J=$ $1.35,1.65,1.95$, respectively. The radial components of these are given in (d). No attraction is found for these parameter values. 
(a)

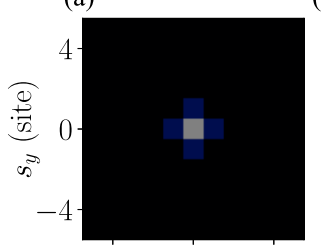

(d)

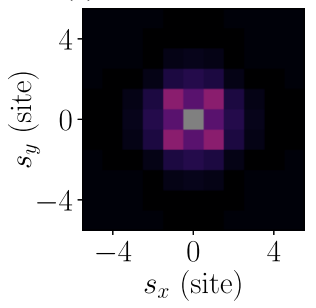

(g)

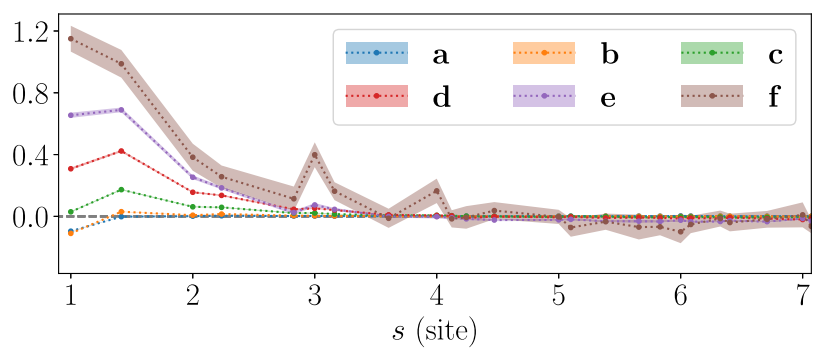

FIG. 12. Hole-hole correlations. (a)-(f) show contour plots of the correlator $C^{\mathrm{h}}(\mathbf{s})$ for the case $t / J=5 / 6$ and $\beta J=$ $0.90,1.50,2.10,2.70,3.30,3.90$, respectively. The radial components of these are given in $(\mathrm{g})$. Attraction is present for all of these parameter values. (b)

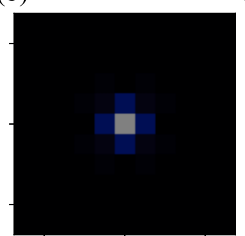

(e)

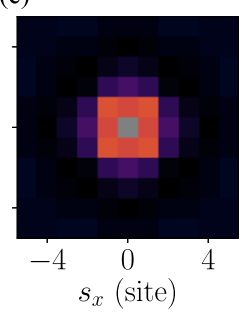

(f)
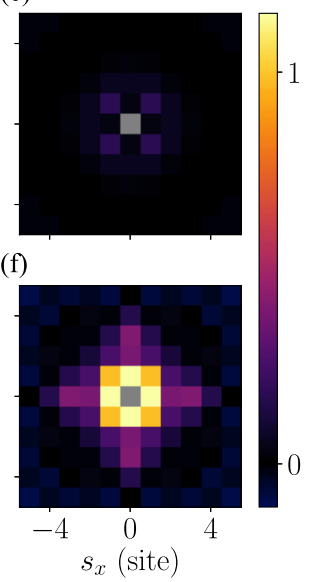

ob

. observed.

Due to the presence of the fermionic sign problem, the noise grows exponentially with an increased value of $t \beta$ and $J \beta$. Data at these parameter values, therefore, suffer from a poor SNR. Data at small values of $t \beta$ and $J \beta$ also have a low SNR, but here the reason being a weak signal. To filter out noisy data, the SNR is defined as $\left(C_{\max }-C_{\min }\right)^{2} /\left\langle n^{2}\right\rangle$, i.e., the ratio of squared signal amplitude to mean square noise. A data point is deemed too noisy and ignored if SNR $<200$.

To reduce noise, the outer edges of the background correlation, where $s_{x}, s_{y}=10$ (we performed simulations on systems with $20 \times 20$ lattice sites), are omitted. These omitted correlation values merely carry half, or even a quarter, of the statistical weight compared to other background values. The SNR of the remaining correlator is, therefore, improved.
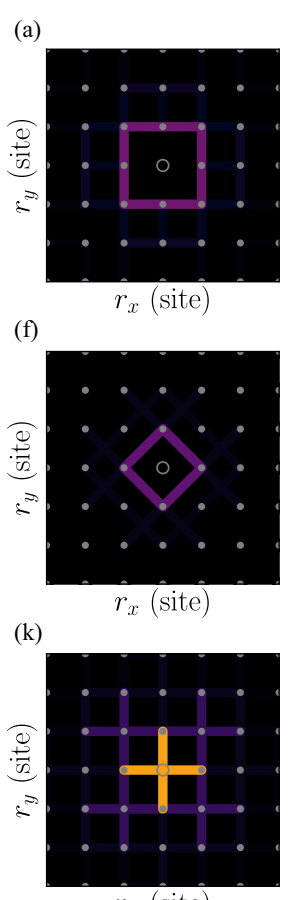

$r_{x}$ (site) (b)
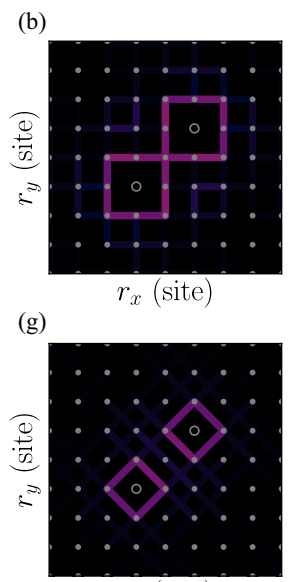

(1)

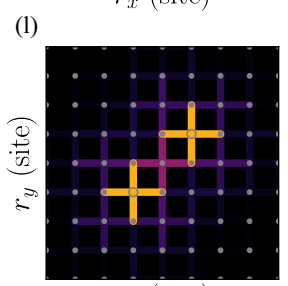

$r_{x}$ (site)

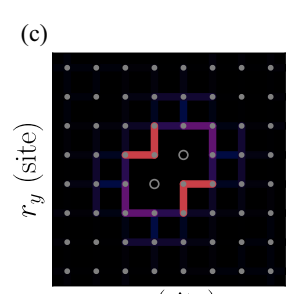

(h)

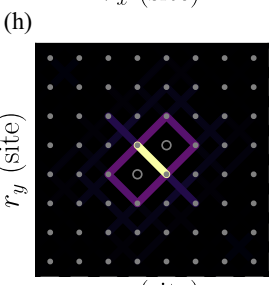

(m)

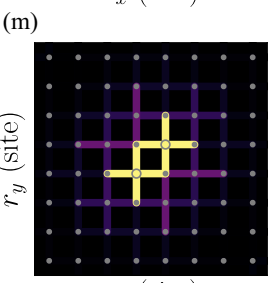

$r_{x}$ (site)
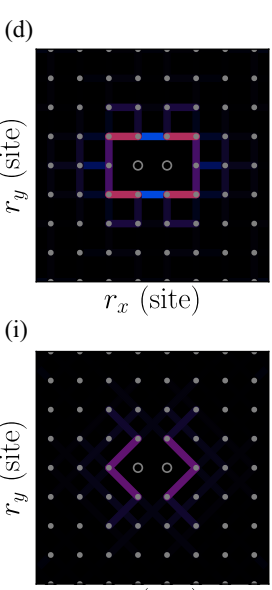

$r_{x}$ (site)

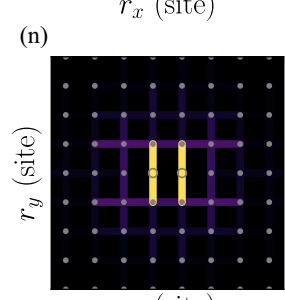

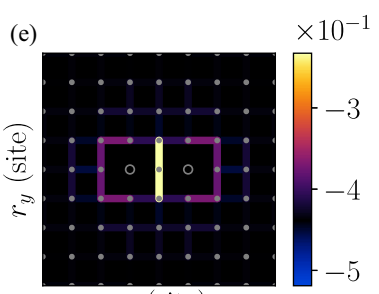
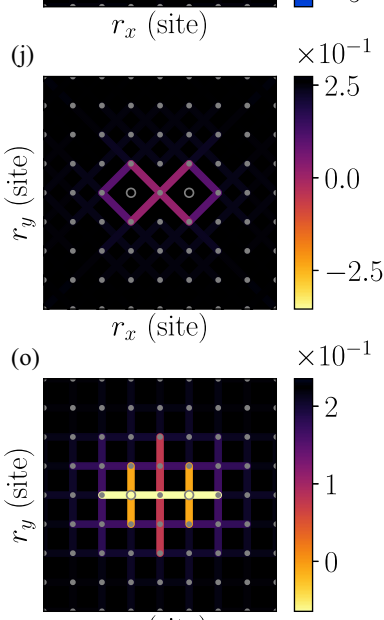

FIG. 13. Spin correlations $C_{|\mathbf{d}|, \mathbf{s}}^{\mathrm{s}}(\mathbf{r})$. The left column depicts a single hole, while the remaining columns depict two dopants located in the close vicinity of one another. The top row (a)-(e) gives $C_{|\mathbf{d}|=1, \mathbf{s}}^{\mathrm{s}}(\mathbf{r})$, middle row (f)-(j) gives $C_{|\mathbf{d}|=\sqrt{2}, \mathbf{s}}^{\mathrm{s}}(\mathbf{r})$, and bottom row (k)-(o) gives $C_{|\mathbf{d}|=2, \mathbf{s}}^{\mathrm{s}}(\mathbf{r})$. Model parameters are $t \beta=2.75$ and $J \beta=3.3$. 

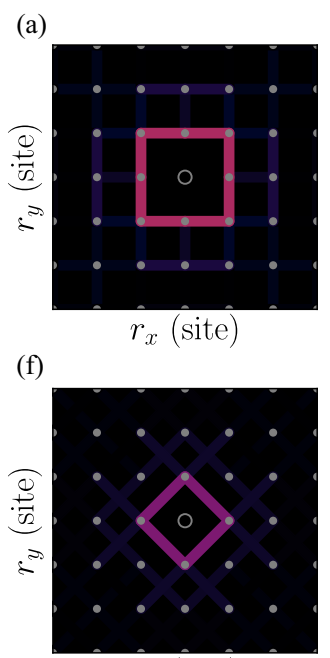

$r_{x}$ (site)

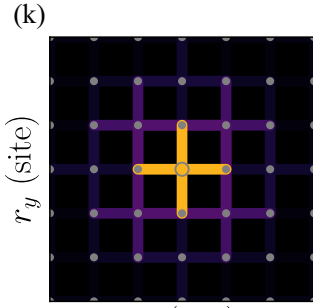

$r_{x}$ (site)

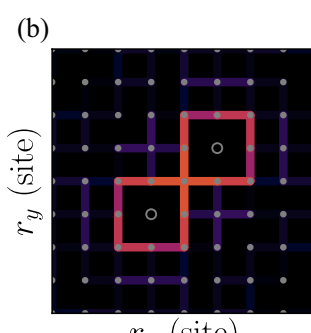

(g)

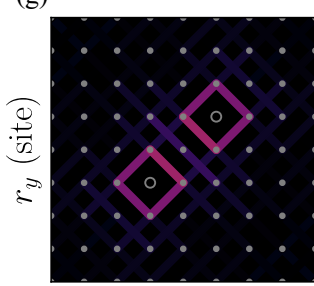

$r_{x}$ (site)

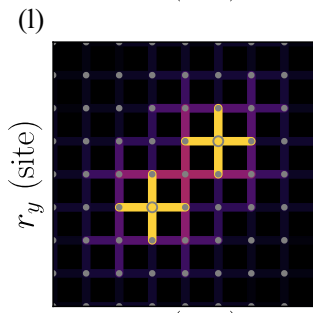

$r_{x}$ (site)

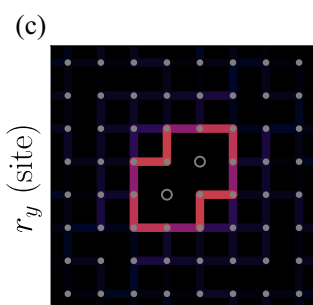

$r_{x}$ (site)

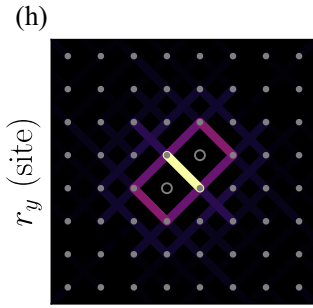

$r_{x}$ (site)

(m)

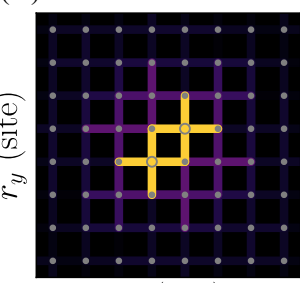

$r_{x}$ (site)

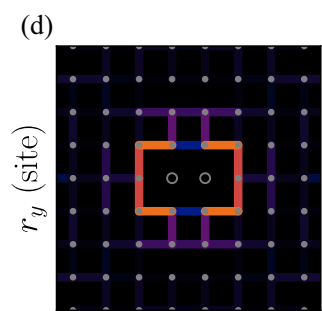

$r_{x}$ (site)

(i)

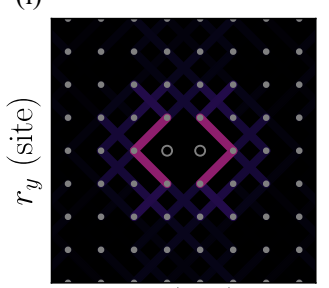

$r_{x}$ (site)

(n)

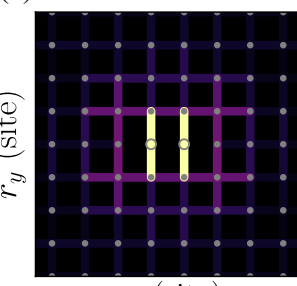

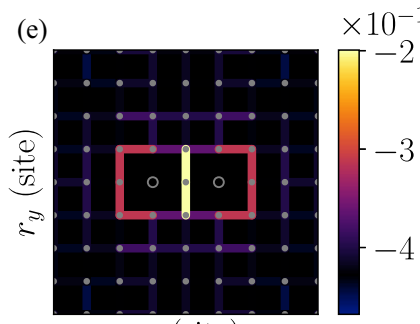

$r_{x}$ (site)

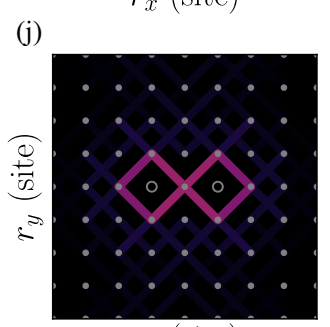

$\times 10^{-1}$

$-2.5$

$-0.0$

$-2.5$

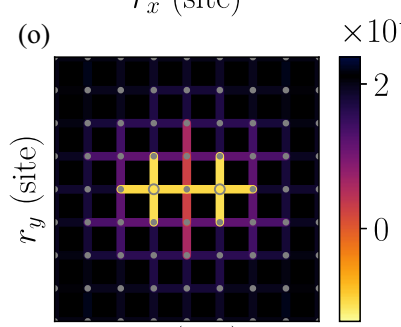

$r_{x}$ (site)

FIG. 14. Spin correlations $C_{|\mathbf{d}| \mathbf{s}}^{\mathrm{s}}(\mathbf{r})$. The left column depicts a single hole, while the remaining columns depict two dopants located in the close vicinity of one another. The top row (a)-(e) gives $C_{|\mathbf{d}|=1, \mathbf{s}}^{\mathrm{s}}(\mathbf{r})$, middle row (f)-(j) gives $C_{|\mathbf{d}|=\sqrt{2}, \mathbf{s}}^{\mathrm{s}}(\mathbf{r})$, and bottom row (k)-(o) gives $C_{|\mathbf{d}|=2, \mathbf{s}}^{\mathrm{s}}(\mathbf{r})$. Model parameters are $t \beta=4.5$ and $J \beta=2.7$.

(a)
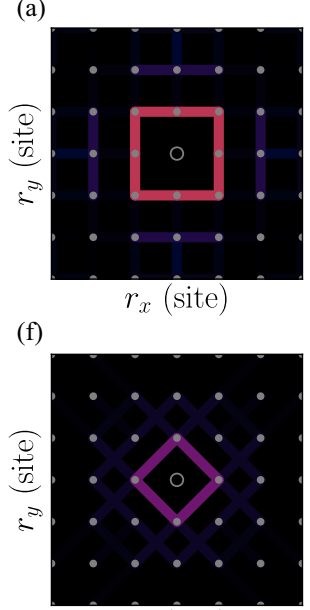

(k)

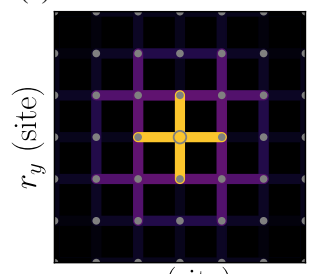

(b)

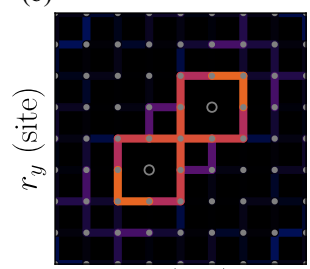

$r_{x}$ (site)

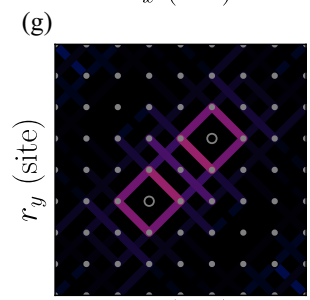

$r_{x}$ (site)

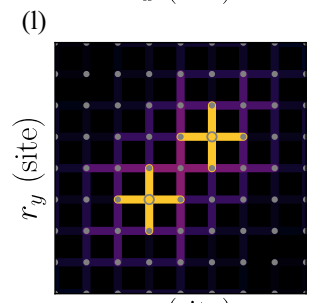

$r_{x}$ (site) (c)

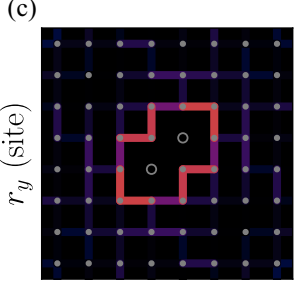

$r_{x}$ (site)

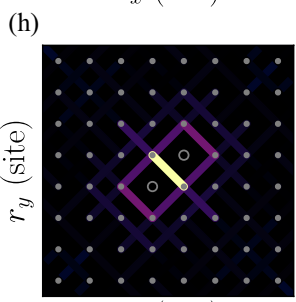

$r_{x}$ (site)

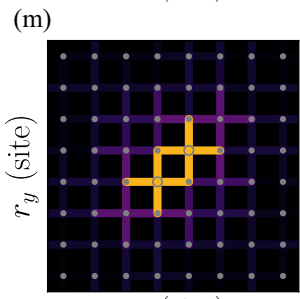

$r_{x}$ (site)

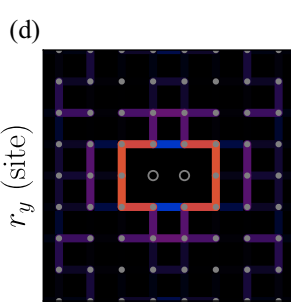

$r_{x}$ (site)

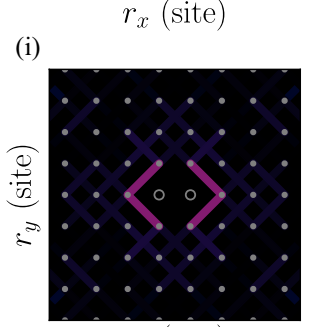

$r_{x}$ (site)

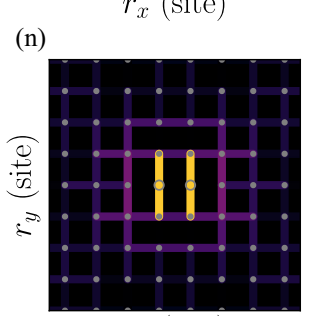

$r_{x}$ (site)

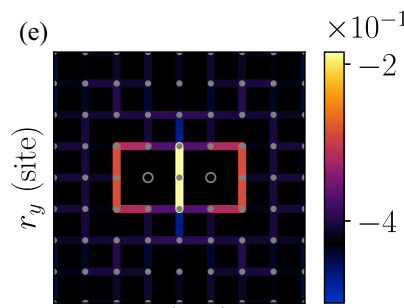

$r_{x}$ (site)

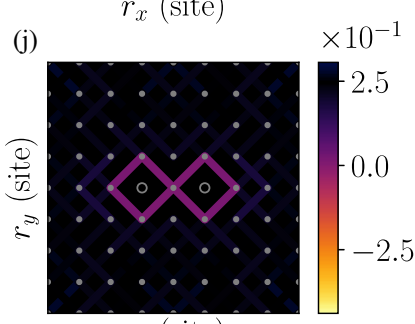

$r_{x}$ (site)

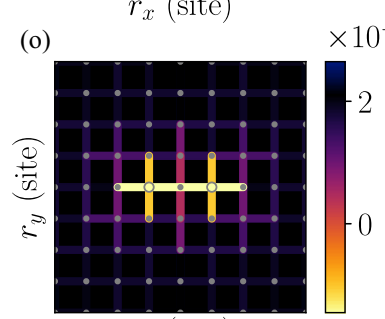

$r_{x}$ (site)

FIG. 15. Spin correlations $C_{|\mathbf{d}|, \mathbf{s}}^{\mathrm{s}}(\mathbf{r})$. The left column depicts a single hole, while the remaining columns depict two dopants located in the close vicinity of one another. The top row (a)-(e) gives $C_{|\mathbf{d}|=1, \mathbf{s}}^{\mathrm{s}}(\mathbf{r})$, middle row (f)-(j) gives $C_{|\mathbf{d}|=\sqrt{2}, \mathbf{s}}^{\mathrm{s}}(\mathbf{r})$, and bottom row (k)-(o) gives $C_{|\mathbf{d}|=2, \mathbf{s}}^{\mathrm{s}}(\mathbf{r})$. Model parameters are $t \beta=5.4$ and $J \beta=2.7$. 
To further improve SNR, we ultimately performed largescale simulations, amounting to several million core-hours. The number of sampled world-line configurations for different values of $t \beta$ and $J \beta$ is shown in Fig. 10.

\section{Additional hole-hole correlation data}

In Figs. 11 and 12, we present additional data of the holehole correlator defined in Eq. (A1).

\section{Additional spin-spin correlation data}

In Figs. 13-15, we present additional data of the spin-spin correlator, defined by

$$
C_{|\mathbf{d}| \mathbf{s}}^{\mathrm{s}}(\mathbf{r})=4 \frac{\sum_{\mathbf{r}_{0}}\left\langle\hat{n}_{\mathbf{r}_{0}}^{\mathrm{h}} \hat{n}_{\mathbf{r}_{0}+\mathbf{s}}^{\mathrm{h}} \hat{S}_{\mathbf{r}_{0}+\mathbf{r}-\mathbf{d} / 2}^{z} \hat{S}_{\mathbf{r}_{0}+\mathbf{r}+\mathbf{d} / 2}^{z}\right\rangle}{\sum_{\mathbf{r}_{0}}\left\langle\hat{n}_{\mathbf{r}_{0}}^{\mathrm{h}} \hat{n}_{\mathbf{r}_{0}+\mathbf{s}}^{\mathrm{h}}\right\rangle} .
$$

Further examples are given as animations [46].

\section{Exact diagonalization}

In order to verify the accuracy of WAMC, it is been benchmarked against exact diagonalization (ED) [49] on a system

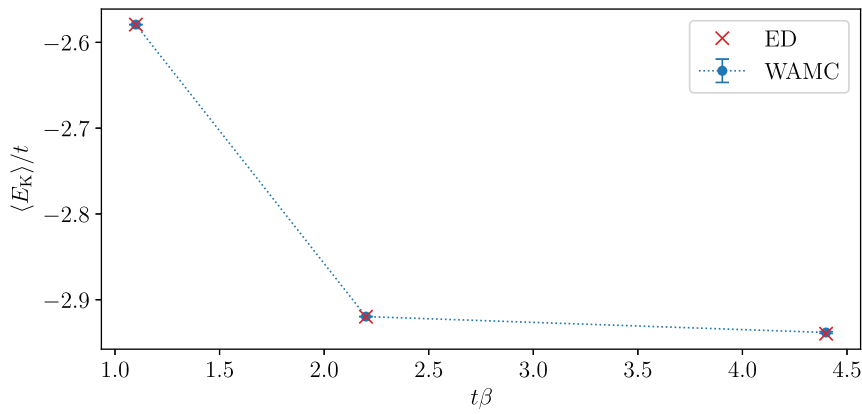

FIG. 16. Kinetic energy. A single carrier's average kinetic energy, as a function of temperature, in a system of $4 \times 4$ lattice sites with periodic boundary conditions. The ratio of hopping strength to super-exchange strength is $t / J=10 / 3$. While error bars are given for the WAMC data, they are smaller than the marker size. The data indicate a perfect agreement between WAMC and ED.

of $4 \times 4$ lattice sites with periodic boundaries, containing a single carrier. In Fig. 16 we present average kinetic energy results, which indicate a perfect agreement between the unbiased WAMC and exact ED methods.
[1] P. A. Lee, N. Nagaosa, and X.-G. Wen, Doping a Mott insulator: Physics of high-temperature superconductivity, Rev. Mod. Phys. 78, 17 (2006).

[2] J. Koepsell, D. Bourgund, P. Sompet, S. Hirthe, A. Bohrdt, Y. Wang, F. Grusdt, E. Demler, G. Salomon, C. Gross, and I. Bloch, Microscopic evolution of doped Mott insulators from polaronic metal to Fermi liquid, arXiv:2009.04440.

[3] P. W. Anderson, The resonating valence bond state in $\mathrm{La}_{2} \mathrm{CuO}_{4}$ and superconductivity, Science 235, 1196 (1987).

[4] V. J. Emery and S. A. Kivelson, Importance of phase fluctuations in superconductors with small superfluid density, Nature (London) 374, 434 (1995).

[5] V. B. Geshkenbein, L. B. Ioffe, and A. I. Larkin, Superconductivity in a system with preformed pairs, Phys. Rev. B 55, 3173 (1997).

[6] M. A. Kastner, R. J. Birgeneau, G. Shirane, and Y. Endoh, Magnetic, transport, and optical properties of monolayer copper oxides, Rev. Mod. Phys. 70, 897 (1998).

[7] M. S. Hybertsen, E. B. Stechel, M. Schluter, and D. R. Jennison, Renormalization from density-functional theory to strong-coupling models for electronic states in $\mathrm{Cu}-\mathrm{O}$ materials, Phys. Rev. B 41, 11068 (1990).

[8] J. P. F. LeBlanc, A. E. Antipov, F. Becca, I. W. Bulik, G. K.-L. Chan, C.-M. Chung, Y. Deng, M. Ferrero, T. M. Henderson, C. A. Jiménez-Hoyos et al. (Simons Collaboration on the Many-Electron Problem), Solutions of the Two-Dimensional Hubbard Model: Benchmarks and Results from a Wide Range of Numerical Algorithms, Phys. Rev. X 5, 041041 (2015).

[9] S. R. White, Density Matrix Formulation for Quantum Renormalization Groups, Phys. Rev. Lett. 69, 2863 (1992).

[10] W. Metzner and D. Vollhardt, Correlated Lattice Fermions in $d=\infty$ Dimensions, Phys. Rev. Lett. 62, 324 (1989).
[11] A. Toschi, A. A. Katanin, and K. Held, Dynamical vertex approximation: A step beyond dynamical mean-field theory, Phys. Rev. B 75, 045118 (2007).

[12] A. N. Rubtsov, M. I. Katsnelson, and A. I. Lichtenstein, Dual fermion approach to nonlocal correlations in the Hubbard model, Phys. Rev. B 77, 033101 (2008).

[13] S.-X. Yang, H. Fotso, S.-Q. Su, D. Galanakis, E. Khatami, J.-H. She, J. Moreno, J. Zaanen, and M. Jarrell, Proximity of the Superconducting Dome and the Quantum Critical Point in the Two-Dimensional Hubbard Model, Phys. Rev. Lett. 106, 047004 (2011).

[14] E. Gull, O. Parcollet, and A. J. Millis, Superconductivity and the Pseudogap in the Two-Dimensional Hubbard Model, Phys. Rev. Lett. 110, 216405 (2013).

[15] T. A. Maier, M. Jarrell, T. Prushke, and M. Hettler, Quantum cluster theories, Rev. Mod. Phys. 77, 1027 (2005).

[16] S. R. White, D. J. Scalapino, R. L. Sugar, N. E. Bickers, and R. T. Scalettar, Attractive and repulsive pairing interaction vertices for the two-dimensional Hubbard model, Phys. Rev. B 39, 839 (1989).

[17] J. E. Hirsch and S. Tang, Antiferromagnetism in the TwoDimensional Hubbard Model, Phys. Rev. Lett. 62, 591 (1989).

[18] S. R. White, D. J. Scalapino, R. L. Sugar, E. Y. Loh, J. E. Gubernatis, and R. T. Scalettar, Numerical study of the twodimensional Hubbard model, Phys. Rev. B 40, 506 (1989).

[19] S. Zhang, Auxiliary-field quantum Monte Carlo for correlated electron systems, in Emergent Phenomena in Correlated Matter Modeling and Simulation Vol. 3 (Forschungszentrum Jülich, Jülich, 2013).

[20] D. J. Scalapino, Numerical studies of the 2D Hubbard model, Handbook of High-Temperature Superconductivity, edited by J. R. Schrieffer and J. S. Brooks (Springer, New York, NY, 2007). 
[21] M. Rigol, T. Bryant, and R. R. P. Singh, Numerical LinkedCluster Approach to Quantum Lattice Models, Phys. Rev. Lett. 97, 187202 (2006).

[22] E. Khatami, E. Perepelitsky, M. Rigol, and B. S. Shastry, Linked-cluster expansion for the Green's function of the infinite- $U$ Hubbard model, Phys. Rev. E 89, 063301 (2014).

[23] K. V. Houcke, E. Kozik, N. Prokof'ev, and B. Svistunov, Diagrammatic Monte Carlo, Phys. Proc. 6, 95 (2010), special issue, Computer Simulations Studies in Condensed Matter Physics XXI.

[24] Y. Deng, E. Kozik, N. V. Prokof'ev, and B. V. Svistunov, Emergent BCS regime of the two-dimensional fermionic Hubbard model: Ground-state phase diagram, Europhys. Lett. 110, 57001 (2015).

[25] J. Carlström, Spin-charge transformation of lattice fermion models: Duality approach for diagrammatic simulation of strongly correlated systems, J. Phys.: Condens. Matter 29, 385602 (2017).

[26] J. Carlström, Diagrammatic Monte Ccarlo procedure for the spin-charge transformed Hubbard model, Phys. Rev. B 97, 075119 (2018).

[27] J. Carlström, Strong-coupling diagrammatics for lattice fermions and spins based on irreducible vertices, arXiv:1909.00816.

[28] A. J. Kim, N. V. Prokof'ev, B. V. Svistunov, and E. Kozik, Homotopic action: A pathway to convergent diagrammatic theories, arXiv:2010.05301.

[29] F. Šimkovic and E. Kozik, Determinant Monte Carlo for irreducible Feynman diagrams in the strongly correlated regime, Phys. Rev. B 100, 121102(R) (2019).

[30] A. J. Kim, F. Simkovic, and E. Kozik, Spin and Charge Correlations Across the Metal-to-Insulator Crossover in the Half-Filled 2D Hubbard Model, Phys. Rev. Lett. 124, 117602 (2020).

[31] D. Poilblanc, J. Riera, and E. Dagotto, $d$-wave bound state of holes in an antiferromagnet, Phys. Rev. B 49, 12318 (1994).

[32] C. Gross and I. Bloch, Quantum simulations with ultracold atoms in optical lattices, Science 357, 995 (2017).

[33] A. Mazurenko, C. S. Chiu, G. Ji, M. F. Parsons, M. KanászNagy, R. Schmidt, F. Grusdt, E. Demler, D. Greif, and M. Greiner, A cold-atom Fermi-Hubbard antiferromagnet, Nature (London) 545, 462 (2017).

[34] L. W. Cheuk, M. A. Nichols, K. R. Lawrence, M. Okan, H. Zhang, E. Khatami, N. Trivedi, T. Paiva, M. Rigol, and M. W. Zwierlein, Observation of spatial charge and spin correlations in the 2D Fermi-Hubbard model, Science 353, 1260 (2016).
[35] M. F. Parsons, A. Mazurenko, C. S. Chiu, G. Ji, D. Greif, and M. Greiner, Site-resolved measurement of the spin-correlation function in the Fermi-Hubbard model, Science 353, 1253 (2016).

[36] J. Koepsell, J. Vijayan, P. Sompet, F. Grusdt, T. A. Hilker, E. Demler, G. Salomon, I. Bloch, and C. Gross, Imaging magnetic polarons in the doped Fermi-Hubbard model, Nature (London) 572, 358 (2019).

[37] C. S. Chiu, G. Ji, A. Bohrdt, M. Xu, M. Knap, E. Demler, F. Grusdt, M. Greiner, and D. Greif, String patterns in the doped Hubbard model, Science 365, 251 (2019).

[38] N. V. Prokof'ev, B. V. Svistunov, and I. S. Tupitsyn, Exact, complete, and universal continuous-time worldline Monte Carlo approach to the statistics of discrete quantum systems, J. Exp. Theor. Phys. 87, 310 (1998).

[39] A. S. Mishchenko, N. V. Prokof'ev, and B. V. Svistunov, Singlehole spectral function and spin-charge separation in the $t-J$ model, Phys. Rev. B 64, 033101 (2001).

[40] E. Blomquist and J. Carlström, Unbiased description of magnetic polarons in a Mott insulator, Commun. Phys. 3, 172 (2020).

[41] J. Spałek, $t-J$ model then and now: A personal perspective from the pioneering times, Acta Phys. Pol. A 111, 409 (2007).

[42] J. Hubbard, Electron correlations in narrow energy bands, Proc. R. Soc. London A 276, 238 (1963).

[43] E. Dagotto, Correlated electrons in high-temperature superconductors, Rev. Mod. Phys. 66, 763 (1994).

[44] S. R. White and D. J. Scalapino, Hole and pair structures in the t-J model, Phys. Rev. B 55, 6504 (1997).

[45] F. Grusdt, A. Bohrdt, and E. Demler, Microscopic spinonchargon theory of magnetic polarons in the $t-J$ model, Phys. Rev. B 99, 224422 (2019).

[46] E. Blomquist, Hole-hole and spin-spin correlations in the $t$ $J$ model (unpublished), https://www.youtube.com/watch?v=9f0qDY1UTU.

[47] K. Sellin, Structure formation, phase transitions and drag interactions in multicomponent superconductors and superfluids, Ph.D. thesis, KTH, Statistical Physics, 2018.

[48] M. Troyer and U.-J. Wiese, Computational Complexity and Fundamental Limitations to Fermionic Quantum Monte Carlo Simulations, Phys. Rev. Lett. 94, 170201 (2005).

[49] A. W. Sandvik, Computational studies of quantum spin systems, in Lectures on the Physics of Strongly Correlated Systems XIV: Fourteenth Training Course in the Physics of Strongly Correlated Systems, 5-16 October 2009, Vietri sul Mare, Italy, edited by A. Avella and F. Mancini, AIP Conf. Proc. No. 1297 (AIP, New York, 2010), p. 135. 